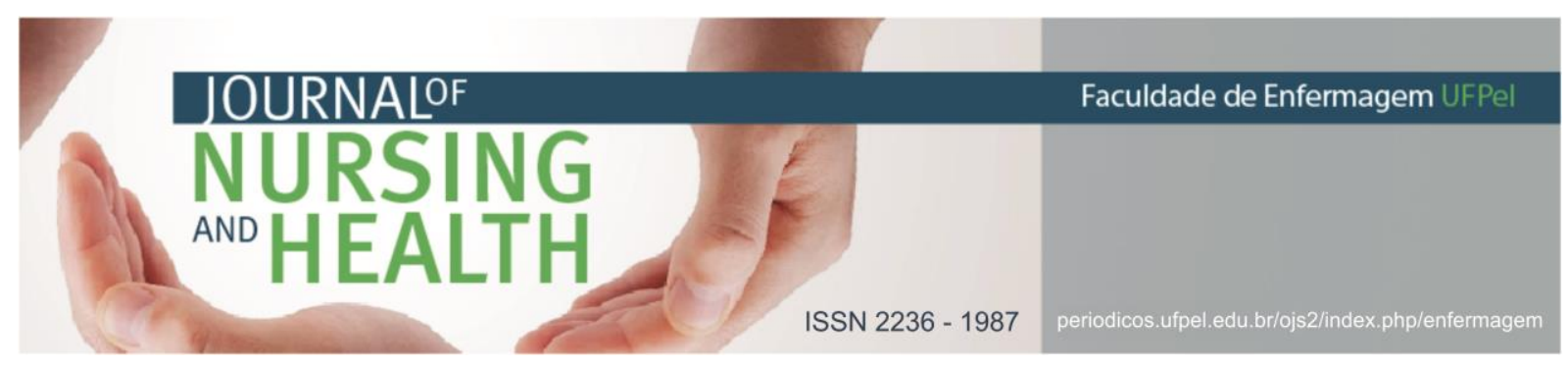

ARTIGO ORIGINAL

\title{
Perfil epidemiológico da hanseníase em Itabuna - Bahia
}

\author{
Epidemiological profile of leprosy in Itabuna - Bahia \\ Perfil Epidemiológico de la Lepra en Itabuna - Bahia \\ Santana, Joyce Costa de ${ }^{1}$; Santos, Mayssa Carvalho²; Lima, Márcio Amorim Tolentino³; Carvalho, \\ Lucas Ribeiro de ${ }^{4}$
}

Como citar este artigo: Santana JC, Santos C, Lima MA, Carvalho LR. Perfil Epidemiológico da hanseníase em Itabuna - Bahia. J. nurs. health. 2018;8(2):e188206

\section{RESUMO}

Objetivo: analisar o perfil epidemiológico dos novos registros de hanseníase na cidade de Itabuna, Bahia no período de 2010 a 2014. Métodos: estudo exploratório, descritivo e quantitativo, através da coleta de dados secundários registrados no Sistema de Informação de Agravos de Notificação. Resultados: houve tendência de decréscimo no número de casos entre 2010 e 2014, predominou o sexo masculino $(56,1 \%)$, a faixa etária entre 50 a 64 anos $(23,5 \%)$, cor da pele parda $(58,7 \%)$, ensino fundamental incompleto (40,2\%) Conclusão: a Hanseníase em Itabuna-BA ocorre mais comumente em adultos e idosos, prevalecendo indivíduos com mais de 50 anos, de cor de pele parda e com baixa escolaridade, indicando pessoas que geralmente são economicamente ativas e com pouco acesso à informação.

Descritores: Epidemiologia; Hanseníase; Incidência

\section{ABSTRACT}

Objective: to analyze the epidemiological profile of the new records of leprosy in the city of Itabuna, Bahia from 2010 to 2014. Methods: exploratory, descriptive and quantitative study was conducted by collecting secondary data recorded in the Information System Notifiable Diseases. Results: there was a decrease in the number of cases between 2010 and 2014, the predominance of males $(56,1 \%)$, age between 50 to 64 years $(23,5 \%)$, brown skin color $(58,7 \%)$, incomplete elementary school $(40,2 \%)$. Conclusions: leprosy in Itabuna-BA occurs most commonly in adults and the elderly, with individuals over 50 years of age, from the brown skin color and with low low schooling, indicating people who are generally economically active and with little access to information.

Descriptors: Epidemiology; Leprosy; Incidence

\footnotetext{
${ }^{1}$ Graduada em Enfermagem. Faculdade do Sul (FACSUL), União Metropolitana de Educação e Cultura (UNIME) Itabuna (BA). E-mail: joycejz@hotmail.com :http://orcid.org/0000-0002-1840-6127

2 Graduada em Enfermagem. Faculdade do Sul (FACSUL), União Metropolitana de Educação e Cultura (UNIME) Itabuna (BA). E-mail: mayssacarvalho1@hotmail.com http://orcid.org/0000-0002-0796-0165

${ }^{3}$ Graduado em Ciências Biológicas. Mestre em sistemas aquáticos tropicais. . Faculdade do Sul (FACSUL). União Metropolitana de Educação e Cultura (UNIME) - Itabuna (BA). E-mail: marcioatl@yahoo.com.br http://orcid.org/0000-0003-1534-8141

${ }^{4}$ Graduado em Biomedicina. Mestre em Biologia e Biotecnologia de Microrganismos. Faculdade do Sul (FACSUL), União Metropolitana de Educação e Cultura (UNIME) E-mail: lucas_carv@hotmail.com http://orcid.org/00000001-5507-6166
} 


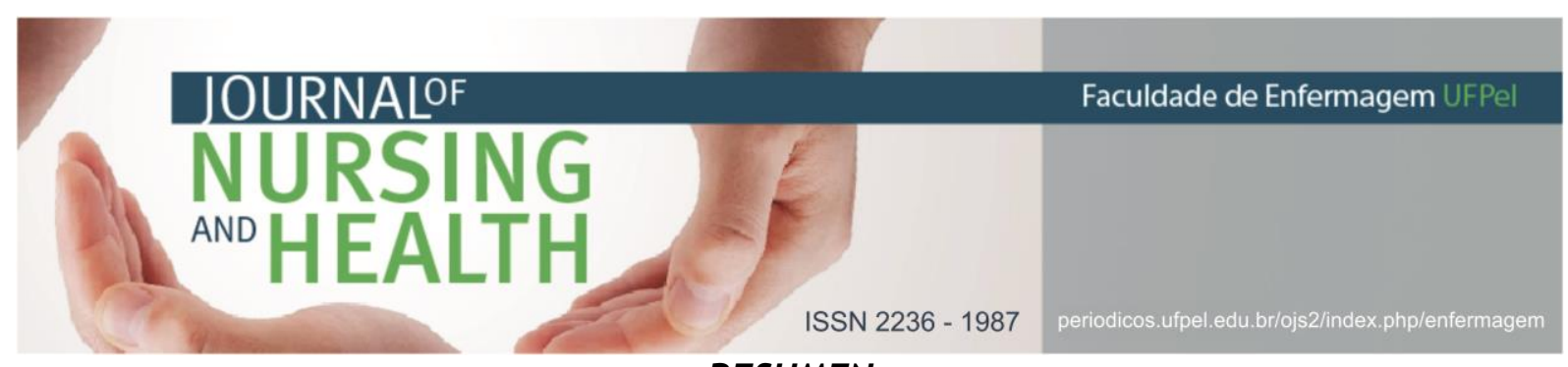

RESUMEN

Objetivo: analizar el perfil epidemiológico de los nuevos registros de lepra en la ciudad de Itabuna, Bahia en el período de 2010 a 2014. Métodos: se realizó un estudio exploratorio, descriptivo y cuantitativo, recolección de datos secundarios registrados en el Sistema de Información de Agravios de Notificación. Resultados: la tendencia de descenso en el número de casos entre 2010 y 2014, el predominio de varones (56.1\%), edad entre 50 a 64 años (23.5\%), color de piel marrón (58.7\%), educación primaria incompleta (40, 2\%). Conclusión: la lepra en Itabuna-BA ocurre con más comúnmente en adultos y ancianos, prevaleciendo individuos con más de 50 años, de color de la piel parda y con baja escolaridad, indicando personas que generalmente son económicamente activas y con poco acceso a la información.

Descriptores: Epidemiología; Lepra; Incidencia

\section{INTRODUÇÃO}

A hanseníase é uma doença infecto-contagiosa crônica que acomete pele e nervos periféricos, causada pelo Mycobacterium leprae. ${ }^{1}$ A principal característica da doença é - comprometimento dos nervos periféricos, isso pode provocar a incapacidade física do indivíduo, inclusive as deformidades e também acarretar problemas psicológicos, limitando assim a vida social dos indivíduos. $^{2}$

A hanseníase é uma das doenças mais antigas registradas e, apesar de existir o tratamento para cura do paciente, ainda é observada no Brasil a notificação de muitos casos, cerca de 2,69/10.000 habitantes, com maior presença crescente nas regiões metropolitanas ${ }^{1,3} 0$ país só perde para a Índia que apresenta mais de $200 \mathrm{mil}$ registros urbanos novos por ano, no surgimento de novos casos em todo o mundo. ${ }^{4-6}$

A transmissão da bactéria ocorre principalmente pelas vias respiratórias superiores e o período de incubação é de 2 a 7 anos, podendo ter períodos mais curtos de 7 meses ou mais longos de 10 anos. ${ }^{7}$ Pessoas de ambos os sexos e em todas as idades podem ser atingidas pela Hanseníase, mas raramente as crianças são infectadas. Em regiões mais pobres e com menor acesso ao saneamento básico, a exemplo do Nordeste do Brasil há maior incidência, observa-se que crianças menores de 15 anos contraem a doença e há uma maior prevalência nos homens do que nas mulheres. ${ }^{8}$

A hanseníase ainda é identificada como um problema de saúde pública no Brasil. Há uma grande variação de coeficiente de prevalência em diferentes regiões do país, devido à situação epidemiológica da doença que é heterogênea. É preciso avaliar o perfil epidemiológico, pois é importante para o controle da quantidade, do crescimento da doença e reduzir a morbidade. Casos novos de hanseníase precisam ser identificados precocemente e feito tratamento para diminuir a transmissão e prevenir a doença. ${ }^{9}$

$\mathrm{Na}$ região do estado da Bahia onde a cidade de Itabuna se localiza, o número de novos registros tem se mostrado contínuo e constante como observado na cidade de vizinha de Ithéus, que no período de 2010 a 2014 apresentou pelo menos 11 casos novos anuais, o que leva a uma preocupação de epidemias da doença, educação 


\section{JOURNALF

sobre a mesma, visto que esta ainda padece de uma elevada carga de preconceitos social e familiar além da ampliação da necessidade das causas desse registro em elevação. ${ }^{10-11}$

Desta forma, o objetivo da pesquisa foi analisar 0 perfil epidemiológico dos casos de hanseníase notificados no período de 2010 a 2014 no município de Itabuna, estado da Bahia.

\section{MATERIAIS E MÉTODOS}

Foi realizado um estudo descritivo, que tem por objetivo determinar a distribuição espacial e/ou temporal de uma doença ou dos seus fatores de risco que atende bem ao que é desejado em pesquisas epidemiológicas, podendo ser realizado a partir de dados primários ou secundários, analisando prevalência ou incidência, bem como os fatores relacionados com a enfermidade observada.

A pesquisa ocorreu no município de Itabuna, localizado no sul do estado da Bahia, na microrregião cacaueira. Limita-se ao Norte com os municípios de Lomanto Júnior e Itajuípe; ao Sul com Jussari e Buerarema; ao Oeste com Itapé e Ibicaraí; e ao Leste com Ilhéus. A área total do município é de $580,49 \mathrm{~km}^{2}$ e sua área urbana é de $65,93 \mathrm{~km}^{2}$. Segundo o último Censo do Instituto Brasileiro de Geografia e Estatística (IBGE) a população está estimada em 218.925 habitantes. ${ }^{12}$

0 objeto de estudo foram os dados secundários, obtidos a partir do Sistema de Informação de Agravos de Notificação (SINAN), dos indivíduos diagnosticados com Hanseníase, em Itabuna-BA, no período de 2010 a 2014.
Por se tratar de uma cidade polo de saúde na microrregião, pacientes dos municípios vizinhos procuram o serviço oferecido na localidade, dado isso, as informações correspondentes aos registros dos usuários dos serviços de saúde que residiam em outros municípios foram excluídas.

As seguintes variáveis foram coletadas: incidência dos casos, sexo, faixa etária, escolaridade, raça, classificação operacional e formas clínicas.

Os dados foram coletados na Vigilância Epidemiológica do município de Itabuna-BA, com acesso ao arquivo eletrônico do SINAN, disponível pelo Departamento de Informática do Sistema Único de Saúde (DATASUS).

A coleta dos dados foi solicitada por meio de oficio à Coordenadora responsável pela Vigilância Epidemiológica do município de Itabuna-BA. Após a autorização, os dados foram coletados em arquivo eletrônico e tabulados em Excel, para elaboração dos gráficos e tabelas. Em relação às considerações éticas, os dados foram copiados na íntegra e com confiabilidade, respeitando as informações que foram fornecidas pelo SINAN e pela Vigilância Epidemiológica do Município com conferência posterior pela cedente dos dados.

\section{RESULTADOS}

Ao longo do período estudado foram registrados no município de Itabuna-BA, 264 casos de Hanseníase, notificados no SINAN: 69 casos em 2010, 58 casos em 2011, 47 casos em 2012, 44 casos em 2013 e 46 casos em 2014. Avaliando a incidência dos casos da doença verificou-se no ano de 2010 
uma maior notificação e uma diminuição no número de casos notificados, a partir de 2011 conforme a Figura 1.

Em relação a variável sexo, nos anos de 2010 a 2014, foram notificados $148(56,1 \%)$ casos do sexo masculino e $116(43,9 \%)$ casos do sexo feminino, demonstrando assim uma leve predominância nos homens.

A análise por ano identificou que em 2010 foram notificados 38 casos do sexo masculino e 31 casos do sexo feminino. No ano de 2011 houve uma igualdade entre os dois sexos com 29 casos. No ano de 2012 foram 31 casos do sexo masculino e 16 casos do sexo feminino. Houve uma aproximação na quantidade dos casos notificados em 2013, com 23 casos do sexo masculino e 21 casos do sexo feminino. E no ano de 2014 foram notificados 27 casos do sexo masculino e 19 casos do sexo feminino.

Em relação à faixa etária a maior frequência foi de 50-64 anos com 62 $(23,5 \%)$ casos, em seguida houve uma igualdade nas faixas etárias de 20-34 anos e 35-49 anos com 60 (22,7\%) casos cada, a faixa etária de 65-79 anos apresentou $35(13,3 \%)$ casos, a faixa etária de 15-19 anos teve 15 (5,7\%) casos e a faixa etária de 80 anos ou mais teve $13(4,9 \%)$ casos. A frequência de indivíduos na faixa etária de 5-9 anos e 10-14 anos foi semelhante com $9(3,4 \%)$ casos notificados em cada. Houve apenas $1(0,4 \%)$ caso notificado na faixa etária de 1-4 anos no ano de 2012.

Ainda com relação à faixa etária observou-se que em 2010 e 2011 a maior frequência ocorreu entre 20-34 anos. Já em 2012 houve mais casos com
50-64 anos. No ano de 2013 os casos foram mais frequentes dos 35-49 anos e 50-64 anos, e no ano de 2014 na faixa etária dos 35-49 anos.

Quanto a cor da pele, a parda foi a mais frequente no período estudado com $155(58,7 \%)$ casos, seguido da raça branca com $51(19,3 \%)$ casos, da raça negra com $46(17,4 \%)$ casos e da raça amarela com 5 (1,9\%) casos. Assim como para a variável escolaridade, também não houve notificação quanto à raça em $7(2,7 \%)$ casos. Avaliando a distribuição das raças dentro dos anos analisados percebeu-se que a cor parda foi a mais frequente em todos os anos, e em 2011 apresentou uma maior quantidade com 40 casos.

$\mathrm{Na}$ classificação operacional, de acordo com o período estudado, foram notificados $149 \quad(56,4 \%)$ casos de Hanseníase multibacilar e 115 (43,6\%) casos de Hanseníase paucibacilar, com uma maior frequência de casos de Hanseníase multibacilar em todos os anos avaliados como demonstrado na Figura 2.

Em relação às formas clinicas, nos anos de 2010 a 2014, foi registrada uma maior incidência da forma Dimorfa com $96(36,3 \%)$ casos. Seguido da forma Tuberculóide com 81 (30,7\%) casos, da forma Virchowiana com $47 \quad(17,8 \%)$ casos e Indeterminada com 33 (12,5\%) casos. Houve $7(2,7 \%)$ casos não notificados quanto à forma clínica.

Analisando dentro dos anos percebeu-se que em 2010, 2011, 2012 e 2014 a forma clínica mais frequente foi a Dimorfa e no ano de 2013 a forma clínica mais frequente foi a Tuberculide com 18 casos, conforme a Figura 3. 


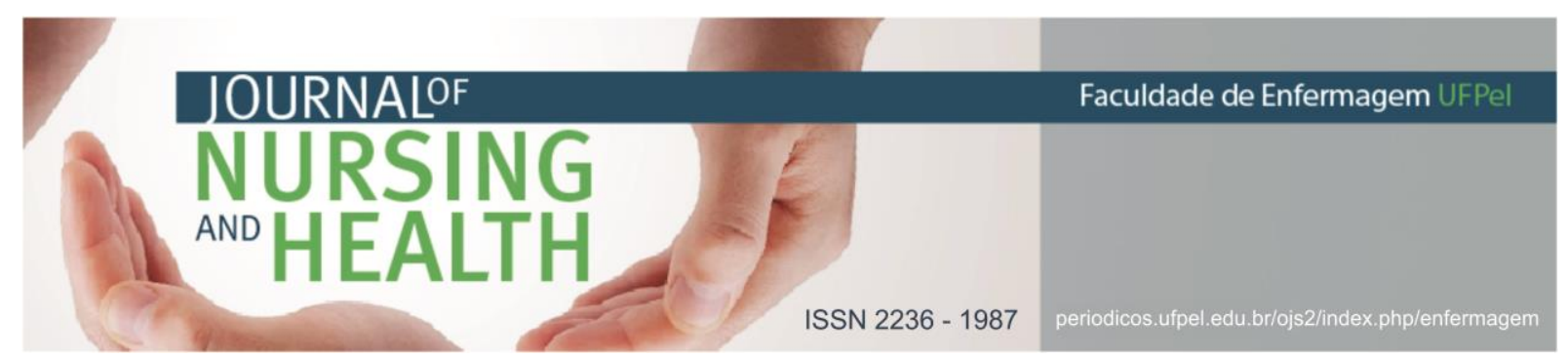

Figura 1: Incidência de casos segundo ano da notificação.

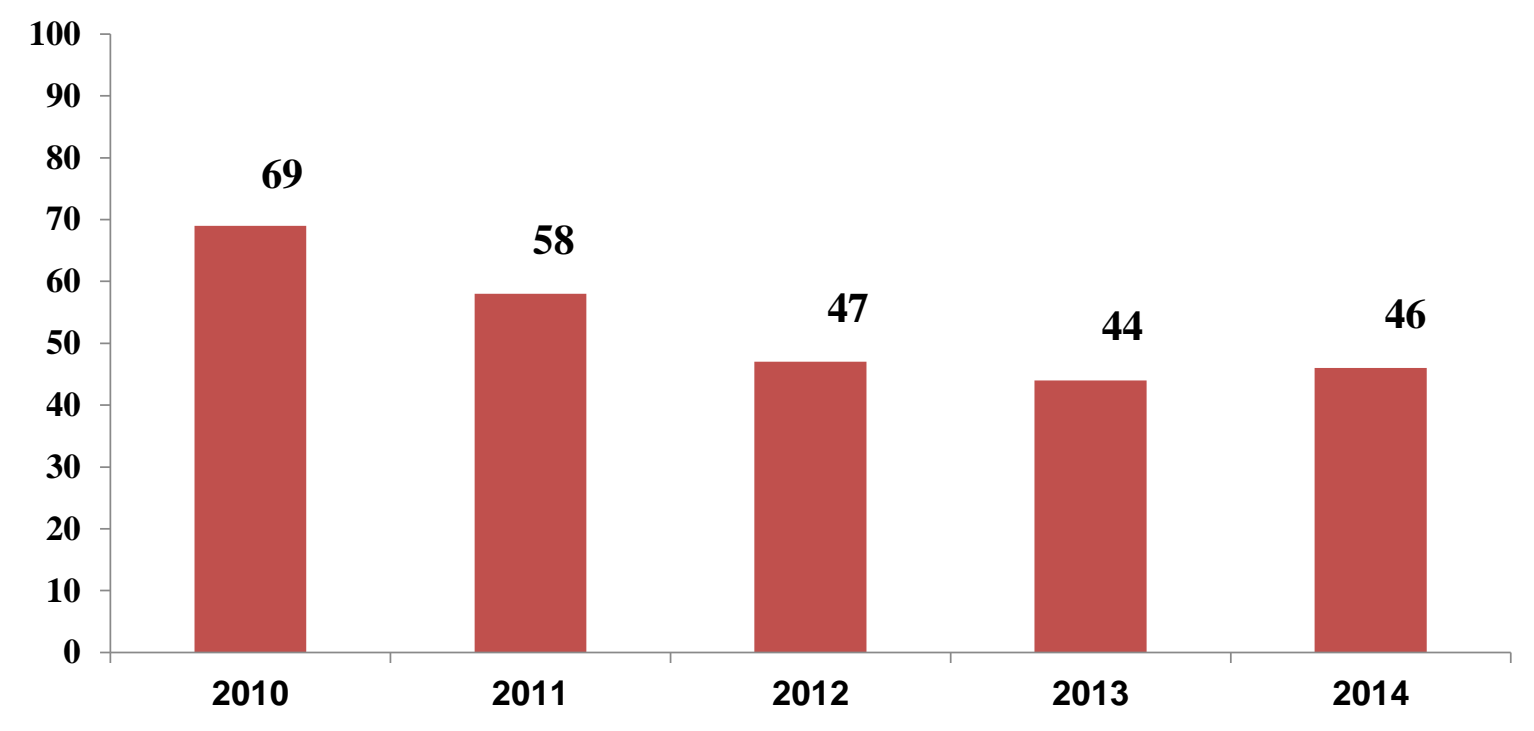

Fonte: SINAN, 2014.

Figura 2: Número de casos por Classificação Operacional segundo ano da notificação.

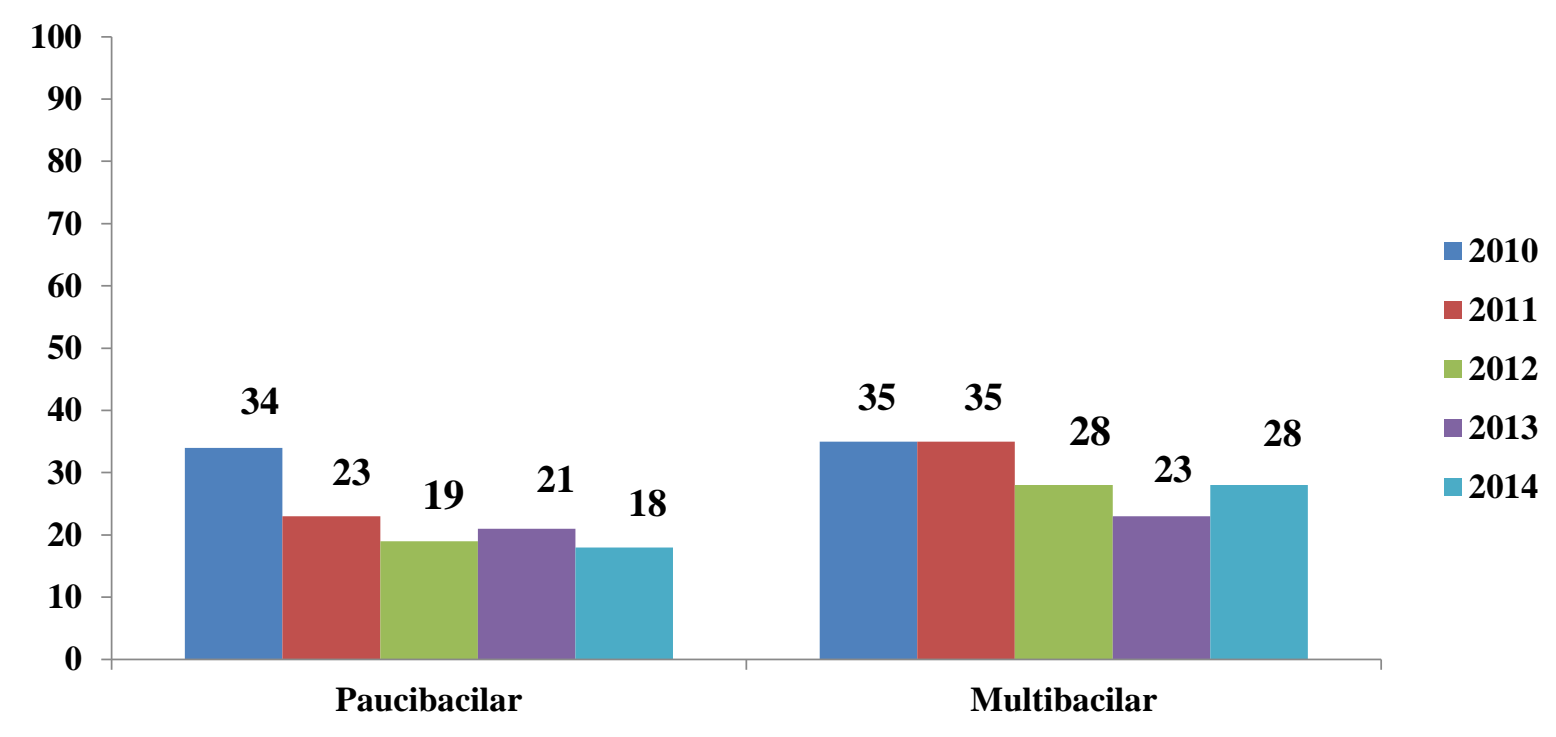

Fonte: SINAN, 2014. 


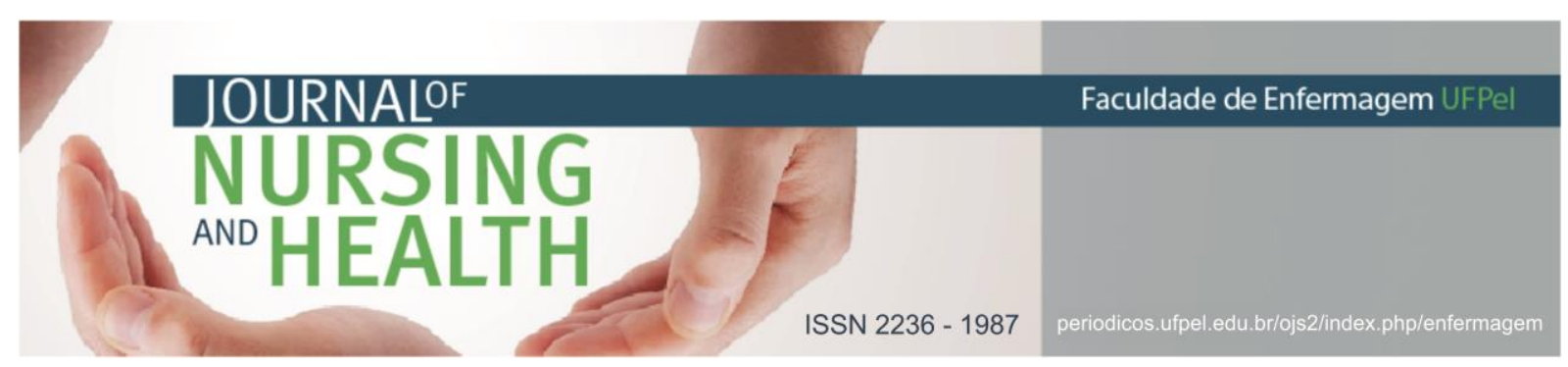

Figura 3: Frequência de casos por forma clínica segundo ano da notificação.

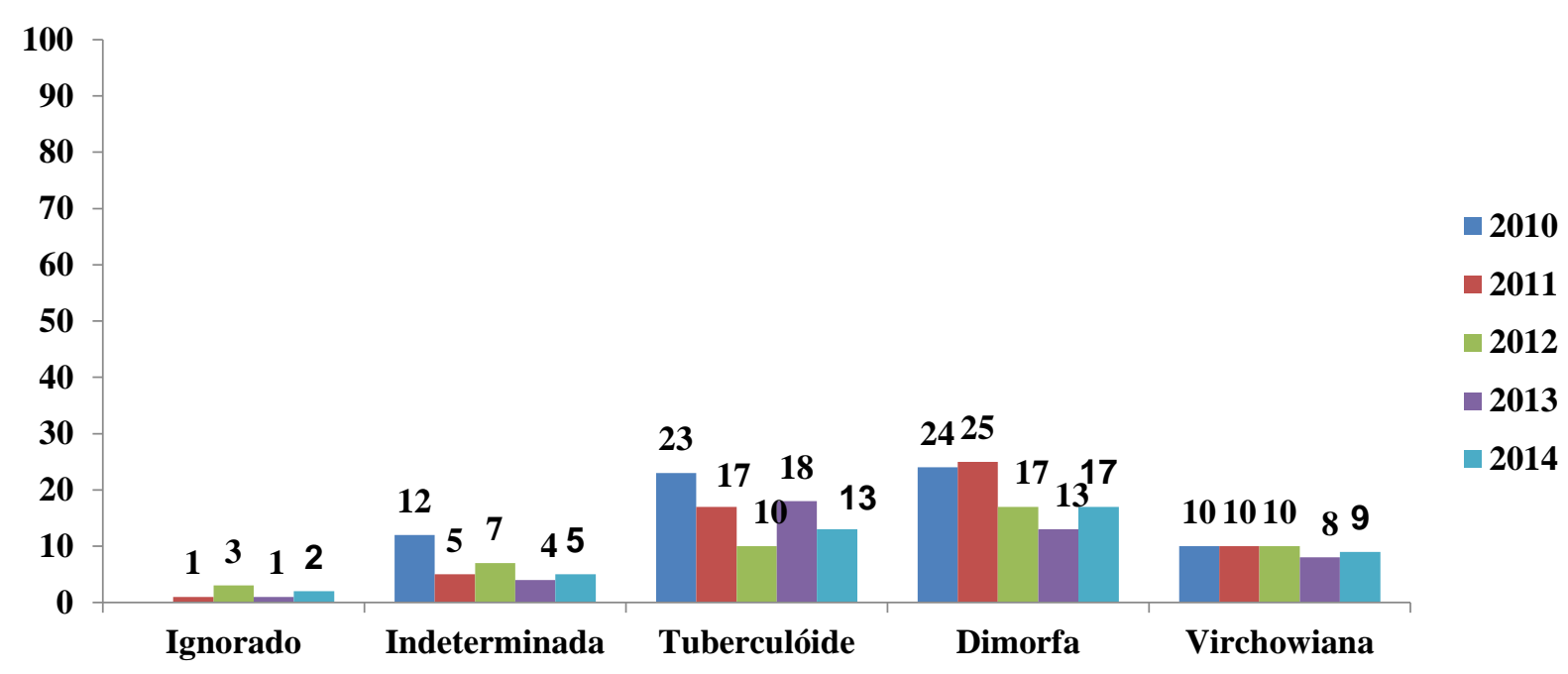

Fonte: SINAN, 2014.

\section{DISCUSSÃO}

Segundo dados oferecidos pelo Ministério da Saúde no período de 2003 a 2013, houve uma diminuição de $68,5 \%$ do coeficiente de prevalência da Hanseníase no Brasil, correspondendo em 2013 um indicador de 1,42 casos/10.000 habitantes, sendo 28.445 casos em tratamento. ${ }^{7}$

Em um estudo do perfil epidemiológico dos casos notificados de Hanseníase no Nordeste brasileiro, ao analisar o coeficiente de detecção geral da doença os pesquisadores verificaram uma estabilidade com uma leve queda nos anos finais na década de 2001-2010. ${ }^{13}$ Como demonstrado no presente estudo em que existe um viés de queda no número de notificações.

No período observado houve uma tendência de redução no número de casos novos com o passar dos anos, porém não há registros de implantação de programas macros pela prefeitura da cidade de Itabuna, que tivessem em seu arcabouço a redução da incidência de hanseníase no período, como o programa nacional Brasil sem miséria, por exemplo.

Quanto à infecção relacionado ao gênero, considera-se que a maior incidência dos casos de Hanseníase, está relacionada ao sexo masculino, pois o homem tem uma preocupação maior com o trabalho e o sustento familiar, o que acaba impedindo-o de procurar as unidades de saúde. Relatase também que os horários de trabalho coincidem com o funcionamento dos serviços de saúde, por isso muitos indivíduos do sexo masculino deixam de buscar atendimento médico. ${ }^{14-15}$

0 padrão observado de jovens adultos e idosos como vítimas mais comuns da hanseníase já foi observado por outros autores que realizaram estudos no estado da Bahia. ${ }^{10,16}$ Esse resultado geralmente é explicado pelo 


\section{JOURNALOF \\ NURSING \\ ANO HEALTH}

ISSN 2236 - 198

longo período de incubação da doença e pelo maior contato social das pessoas durante a vida que aumentam a chance de entrar em um episódio de contágio. ${ }^{16-17}$

Sabe-se que a educação proporciona maior compreensão ao indivíduo no processo saúde-doença, e por isso a frequência de casos de Hanseníase é maior no ensino fundamental incompleto pela falta de informação e conhecimento dos indivíduos, o que acaba influenciando na busca pelo tratamento ${ }^{18}$. Por isso campanhas mais ostensivas e fáceis são necessárias para ajudar a controlar o avanço da doença em regiões endêmicas, visto que a desinformação propicia não só o contágio, mas também o avanço da doença hansênica. ${ }^{2}$

No que se refere a cor da pele, não existem quaisquer correlações entre a pigmentação da pele $\mathrm{e} a$ variação da possibilidade de contágio da hanseníase. 0 resultado diferencial para a cor parda muito se deve à etnia prevalente em uma determinada região, ou como a população local se identifica. ${ }^{18}$ Por se tratar de uma região com predominância de pessoas pardas e negras o resultado encontrado termina estando dentro do esperado para um estudo epidemiológico como em outras áreas do Nordeste. ${ }^{10,16,19}$

No estado de Sergipe, no período de 2001 a 2010, constatou-se que na classificação operacional foram identificados mais casos de Hanseníase multibacilar. Isso significa que a maioria da população apresenta um diagnóstico tardio, contribuindo assim para transmissão de casos multibacilares. ${ }^{13}$ Este fato torna-se preocupante pois nesta forma a doença pode se tornar incapacitante e tornar o tratamento mais complicado. Esse registro pode ser um indicativo também de que o acesso ao diagnóstico é difícil ou o conhecimento da população sobre a doença é baixo ${ }^{15}$ como demonstrado na análise de faixa educacional.

No que se trata da forma clínica, achados semelhantes a esta pesquisa foram encontrados no estudo realizado na Amazônia do Maranhão, em que a forma clínica Dimorfa apresentou maior frequência com $23,3 \%$ do total de 73 casos analisados, ${ }^{20}$ bem como em Ilhéus, cidade vizinha à abordada. ${ }^{10}$

A hanseníase na sua forma dimorfa é conhecida pela sua fácil transmissibilidade e por sua incapacitância, ${ }^{16}$ dado isso, fica clara a necessidade de discussão de novas estratégias das equipes de saúde para impedir o avanço da doença com abordagens mais próximas da população e mais impactantes para permitir que a própria comunidade seja vigia dos hábitos e do possível desenvolvimento da doença em terceiros. ${ }^{20-25}$

\section{CONCLUSÃO}

Os resultados do perfil epidemiológico da hanseníase no município de Itabuna-BA permitiram concluir que houve uma diminuição progressiva na incidência dos casos da doença nos anos de 2010 a 2013 com um pequeno aumento na quantidade de casos em 2014. O sexo masculino apresentou-se mais predominante no período estudado e constatou-se que a doença em Itabuna-BA ocorre com mais frequência em jovens, adultos e 
idosos, da cor parda e com baixa escolaridade.

Em relação à classificação operacional e às formas clínicas, a classe multibacilar e a forma dimorfa foram as mais frequentes, respectivamente. Esses resultados podem colaborar significativamente para compreensão e dimensão da hanseníase no município e, também, fornecer subsídios para o direcionamento de ações de promoção, prevenção e reabilitação da saúde.

\section{REFERÊNCIAS}

1. White C, Franco-Paredes C. Leprosy in the 21st century. Clin microbiology rev [Internet]. 2015[cited 2018 Sept 24];28(1):80-94. Available from: https://www.ncbi.nlm.nih.gov/pmc/a rticles/PMC4284303/

2 Coriolano-Marinus MWL, Pacheco HF, Lima FT, Vasconcelos EMR, Alencar EN. Saúde do escolar: uma abordagem educativa sobre hanseníase. Saúde e transformação social [Internet]. 2012[acesso em 2018 set 24];3(1):72-8. Disponível em: http: / /incubadora.periodicos.ufsc.br/ index.php/saudeetransformacao/artic le/view/1378/1700

3 Magalhães MDCC, Rojas LI. Diferenciação territorial da hanseníase no Brasil. Epidemiol serv saúde [Internet]. 2007[acesso em 2018 set 24];16(2):75-84. Disponível em: http://scielo.iec.gov.br/pdf/ess/v16n 2/v16n2a02.pdf

4 World Health Organization (WHO). Global leprosy: update on the 2012 situation = actualisation de la situation mondiale de la lèpre, 2012. Wkly epidemiol rec [Internet]. 2012[cited
2018 Sept 25];88(35):365-79. Available from:

http: / / apps.who.int/iris/bitstream/h andle/10665/242112/WER8835_365-

379.PDF? sequence $=1 \&$ isAllowed $=y$

5 Lockwood DN, Suneetha S. Leprosy: too complex a disease for a simple elimination paradigm. Health organ bull [Internet]. 2005[cited 2018 Sept 24]; 83(3):230-235. Available from: https://www.ncbi.nlm.nih.gov/pmc/a rticles/PMC2624210/

6 Singal A, Sonthalia S. Leprosy in postelimination era in India: Difficult journey ahead. Indian j dermato [Internet]. 2013[cited 2018 Sept 24];58(6): 443-6. Available from: https://www.ncbi.nlm.nih.gov/pmc/a rticles/PMC3827515/

7 Ministério da Saúde (BR). Secretaria de Vigilância em Saúde. Guia de vigilância em saúde. Brasília; 2014.

8 Freitas BHBMD, Cortela DDCB, Ferreira SMB. Tendência da hanseníase em menores de 15 anos em Mato Grosso (Brasil), 2001-2013. Rev saude publica [Internet]. 2017[acesso em 2018 set 24];51:1-9. Disponível em: http: //www.scielo.br/pdf/rsp/v51/pt _0034-8910-rsp-S1518-

87872017051006884.pdf

9 Pereira MA, Moura LMA, Veloso LC. Estratégias utilizadas pelos serviços de saúde na detecção precoce da hanseníase: uma revisão integrativa. Saúde foco [Internet]. 2015[acesso em 2018 set 24];2(1): 130-50. Disponível em:

http: / /www4.fsanet.com.br/revista/i ndex.php/saudeemfoco/article/view/ $524 / 850$

10 Freitas DV, Xavier SS, Lima MAT. Perfil Epidemiológico da hanseníase no 


\section{JOURNALOF \\ NURSING \\ ${ }^{A N D} \mathrm{HEALTH}$}

município de Ilhéus-BA, no período de 2010 a 2014. Journal of health science [Internet]. 2018[acesso em 2018 set 24];19(4):274-77. Disponível em: http: / /www.pgsskroton.com.br/seer/ index.php/JHealthSci/article/view/50 08

11 Dias CRS, Gomes GL, Rodrigues AS, Assis ADM. Percepção de usuários sobre o preconceito da hanseníase. Rev rene [Internet]. 2012[acesso em 2018 set 24];13(5): 1004-14. Disponível em: http://www.periodicos.ufc.br/rene/a rticle/view/4081/3189

12 Instituto Brasileiro de Geografia e Estatística (IBGE). IBGE cidades e estados [Internet]. 2014 [acesso em 2015 set 30]. Disponível em: http: / /www.cidades.ibge.gov.br/xtra s/perfil.php?lang=\&codmun=291480

13 Oliveira VM, Assis CRD de, Silva KCC. Levantamento epidemiológico da hanseníase no nordeste brasileiro durante o período de 2001-2010. Scire salutis [Internet]. 2013[acesso em 2018 set 24];3(1):16-27. Disponível em: http://www.sustenere.co/journals/in dex.php/sciresalutis/article/view/ESS 2236-9600.2013.001.0002/232

14 Ramos JM, Martínez-Martín M, Reyes $F$, Lemma D, Belinchón I, Gutiérrez F. Gender differential on characteristics and outcome of leprosy patients admitted to a long-term care rural hospital in South-Eastern Ethiopia. Int $\mathrm{j}$ for equity in health. 2012[cited 2018 Sept 24];11(1):1-7. Available from: https://www.ncbi.nlm.nih.gov/pmc/a rticles/PMC3519584/

15 da Penha AAG, de Oliveira JL, Soares JL, Rufino NF, Rocha RPB, Viana MCA. Desafios na adesão ao tratamento da hanseníase segundo enfermeiros da
ISSN 2236 - 1987

atenção primária à saúde. Cadernos de cultura e ciência [Internet]. 2015[acesso em 2018 set 24];14(2): 7582. Disponível em: http://periodicos.urca.br/ojs/index.p $\mathrm{hp} /$ cadernos/article/view/902/pdf_1

16 Silva MEGDC, Souza CDFD, Costa FMD, Carmo RFD. Epidemiological aspects of leprosy in Juazeiro-BA, from 2002 to 2012. An bras dermato [Internet]. 2015[cited 2018 Sept 24];90(6):799-805. Available from: http://www.scielo.br/pdf/abd/v90n6 10365-0596-abd-90-06-0799.pdf

17 Schneider PB, Freitas BHBMD. Tendência da hanseníase em menores de 15 anos no Brasil, 2001-2016.Cad saúde pública (online) [Internet]. 2018[acesso em 2018 set 24];34(3):e00101817. Disponível em: http://www.scielo.br/pdf/csp/v34n3 /1678-4464-csp-34-03-e00101817.pdf

18 Monteiro BR, Pinheiro MGC, Isoldi DMR, Cabral AMF, Simpson CA, Mendes FRP. Hanseníase: enfocando a educação em saúde para o projovem. Rev pesqui cuid fundam (online) [Internet]. 2015[acesso em 2018 set 25];7(5):49-55. Disponível em: http://www.redalyc.org/pdf/5057/50 5750949005.pdf

19 Brito AL, Monteiro LD, Ramos Junior AN, Heukelbach J, Alencar $\mathrm{CH}$. Tendência temporal da hanseníase em uma capital do Nordeste do Brasil: epidemiologia e análise por pontos de inflexão, 2001 a 2012. Rev bras epidemiol [Internet]. 2016[acesso em 2018 set 25];19(1):194-204. Disponível em:

http: / / www.scielo.br/pdf/rbepid/v19 n1/1980-5497-rbepid-19-01-00194.pdf 
20 Moreira AJ, Naves JM, Fernandes LFRM, de Castro SS, Walsh IAP. Ação educativa sobre hanseníase na população usuária das unidades básicas de saúde de Uberaba-MG. Saúde debate. 2014[acesso em 2018 set 25];38(101):234-43. Disponível em: http://www.scielo.br/pdf/sdeb/v38n 101/0103-1104-sdeb-38-101-0234.pdf

21 Reis ACSM, Borges DPL, D'Avila VGFC, Barbosa MS, Ternes YMF, Santiago SB, et al. O cenário de políticas públicas do Brasil diante do quadro das doenças negligenciadas. Saúde \& ciência em ação. 2017[acesso em 2018 set 25];3(1):99-107. Disponível em: http: / / revistas.unifan.edu.br/index.p $\mathrm{hp} /$ RevistalCS/article/viewFile/237/1 79

22 Fonseca IF, Ramos JLS, da Silva SET, de Oliveira MLB, de Lima Garcia C, Cavalcante EGR, et al. Importância do enfermeiro no controle do tratamento da hanseníase: revisão integrativa. Revista e-ciência [Internet]. 2016[acesso em 2018 set 25]];3(2): 97-106. Disponível em: www.revistafjn.com.br/revista/index. php/eciencia/article/download/88/pd f_19

23 Cunha MHCM da, Silvestre MPSA, Silva AR da, Rosário DDS do, Xavier MB. Fatores de risco em contatos intradomiciliares de pacientes com hanseníase utilizando variáveis clínicas, sociodemográficas e laboratoriais. Rev pan-amaz saude [Internet]. 2017 [acesso em 14 ago 2018];8(2):21-8. Disponível em: http://scielo.iec.gov.br/scielo.php?sc ript=sci_arttext\&pid=S2176$62232017000200021 \& \operatorname{lng}=p t$
ISSN 2236 - 1987

24 Macedo CP, Cerqueira MF de F, Poveda V de B. Avaliação do perfil epidemiológico da hanseníase em um município do interior paulista nos anos de 2000 a 2006. Revista eletrônica de enfermagem do vale do paraíba [Internet]. 2017[acesso em 14 ago 2018];1(4):1-13. Disponível em: http://fatea.br/seer3/index.php/REE NVAP/article/view/91.

25 Aguiar PG, Almeida DA, da Silva SDC, Paschoini J. Fatores de manutenção da endemia hansênica e as ações da enfermagem no controle da hanseníase. Revista de iniciação científica da libertas [Internet]. 2014 [acesso em 14 ago 2018];4(1):119-32. Disponível em: http: / /www.libertas.edu.br/revistas/i ndex.php/riclibertas/article/view/51/ 75

Data de submissão: $27 / 05 / 2018$

Data de aceite: 14/08/2018

Data de publicação: 28/09/2018 